IN PRACTICE

\title{
"We don't really have cause to discuss these things, they don't affect us": a collaborative model for developing culturally appropriate sexual health services with the Bangladeshi community of Tower Hamlets
}

\author{
A Beck, A Majumdar, C Estcourt, J Petrak
}

Sex Transm Infect 2005;81:158-162. doi: 10.1136/sti.2004.012195

See end of article for authors' affiliations

Correspondence to

Dr Andrew Beck, The Ambrose King Centre Royal London Hospital Whitechapel, London É 1 1BB, UK; andrew.beck@ bartsandthelondon.nhs.uk

Accepted for publication 5 July 2004
Objective: To identify barriers to accessing sexual health care among the Bangladeshi community of east London and to develop a model of community participation in service development.

Methods: Qualitative study using one to one interviews with sexual health service users plus focus groups in community settings.

Results: 58 people participated in the study, 12 in individual interviews and the remainder in six focus groups. All were of Bangladeshi origin. Four main themes were reported as impacting on access to services; confidentiality concerns, relevance of services to the community, problems with discussing sexual issues, and problems with previous experiences of health promotion. Community values regarding sex outside of marriage were an important underlying factor in participants' responses. Existing sexual health services were seen as culturally insensitive by patients and community groups.

Conclusions: Community based health initiatives among hard to reach ethnic minority groups should use existing networks of statutory and non-statutory groups to benefit from local expertise and relationships. Steering groups composed of members of the local communities served by the clinic can usefully inform service development.
T he National Strategy for Sexual Health and HIV states that there is a need to engage with at-risk and hard to reach groups in the community in order to meet their sexual health needs. ${ }^{1}$ The Race Relations Act also emphasises the requirement for services, including the NHS, to promote racial equality in access to services ${ }^{2}$ and recent papers have highlighted the need for health authorities and primary care trusts to address health inequality issues among ethnic minority patient groups. Cultural, social, and economic variables have been shown to be predictors of access to services, patterns of service use, and prevalence of sexual health problems. ${ }^{3-5}$ In order to facilitate equality of access, sexual health services must develop culturally appropriate models of service provision and health promotion which are based on an understanding of the barriers to access encountered by that particular societal group.

Recognised barriers to accessing health care in general include personal, social, and cultural factors as well as poor knowledge and awareness of issues relating to the particular health problem, ${ }^{6}$ gender issues regarding the provision of services, and difficulties faced by patients for whom English is not a first language. ${ }^{7}$ In the context of sexual health care, older adults in particular have been identified as having unmet needs which may be difficult for them to raise with general practitioners ${ }^{8}$. In some communities a lack of formal education about sexual issues has been found to be a significant barrier to accessing services. ${ }^{9}$ Within Bangladeshi communities socioeconomic deprivation and environmental problems appear to be particular exacerbating factors. ${ }^{9}$ Clinicians' lack of understanding of cultural mores surrounding sexual education, sexual behaviour, and childbirth experiences are reported to be key factors influencing utilisation of health services by Indian immigrants and their families. ${ }^{10}$ These factors are likely to be shared by people from Bangladeshi communities. It is well recognised that lower socioeconomic status groups are less able to access sexual health services, leading to longer duration of infection and greater burden of untreated sexually transmitted infections (STIs). ${ }^{11}$ Cultural and subcultural factors, underpinned by economic and social inequalities, can therefore have marked impact on sexual behaviours and access to treatment. ${ }^{12}$ Tower Hamlets is home to the largest community of people of Bangladeshi origin in the United Kingdom. Twenty per cent of this community live in seriously overcrowded housing, $80 \%$ have an income below the national average, and the community has the highest levels of unemployment, poorest housing, and lowest levels of educational attainment and health in the United Kingdom. ${ }^{13}$ Thirty five per cent of the population served by the local sexual health clinic are of Bangladeshi origin; however, they represent only $6 \%$ of existing service users. Bangladeshi service users have a largely similar pattern of STIs as non-Bangladeshis but Bangladeshi service users are significantly more likely to be referred by their GP indicating that their knowledge of the service is low. ${ }^{14}$

These differences in patterns of uptake of services prompted us to research the social and cultural mechanisms shaping the Bangladeshi community's relationships with the sexual health service. Here we describe our approach to engaging in research in sexual health issues within a community where cultural, social, and spiritual issues impact on engagement with services. We describe a model for community focused research and services, developing ideas previously used in working with this community. ${ }^{15}$ We discuss findings and recommendations from the study which impact on the development of sexual health services.

\section{METHODS}

For the purposes of this study we used the following terms. A culture is "a shared pattern of belief, feeling, and 
adaptation which people carry in their minds." ${ }^{16}$ A subculture can be seen as any group within a larger complex culture who have interests that are different from those of the mainstream culture. In the United Kingdom, the term "ethnic" is taken to mean a mixture of cultural background and racial designation, and an ethnic group can be characterised by the sense of belonging the individuals feel. ${ }^{17} \mathrm{~A}$ "community" can be described as a body of people having common rights, privileges, or interests, or living in the same place under the same laws and regulations.

\section{Participatory approach}

A steering group was established in order to ensure that the research project met the needs of the local community. This group consisted of members of the Brick Lane Youth Development Association (BLYDA), Social Action for Health, The Bangladeshi Drug Rehabilitation Project (NAFAS), the East London Mosque, a Bangladeshi academic from a nearby university and an ethnic minorities specialist from the London East AIDS Network (LEAN). The majority of the group were of Bangladeshi origin and worked and lived locally. Senior medical and psychology staff also attended the group. The imam of the East London Mosque gave his support for the project and provided consultation time to the project over specific issues. The group met to help establish research questions, examine data as they were collected, refine the next stage of research questions, and then examine the analysed data to generate recommendations for the final report. This iterative model ensured that salient issues for the Bangladeshi community were addressed and ensured a sense of community ownership of the results and subsequent initiatives.

\section{Sampling frame}

Interviews and focus groups took place between January and March 2003.

\section{Interviews}

All sexual health service attenders of Bangladeshi origin who attended within the study period were eligible for the interview section of study. Participants for interview were purposively sampled to ensure inclusion of a broad age range of participants, first and second generation Bangladeshis, and an appropriate gender mix. Interview participants were recruited to the study by AM while they were waiting in the sexual health service waiting room for their medical consultation. Interviews were conducted by $\mathrm{AM}$ in a counselling room.

\section{Focus groups}

Focus groups were composed of non-clinic users of Bangladeshi origin living or working in Tower Hamlets. Participants were nominated by members of the steering group or identified by existing community groups. Six focus groups with community workers and non-service using members of community groups were carried out to explore initial hypotheses generated in interviews and to test emerging recommendations for service development. Focus groups comprised between three and 14 participants plus a facilitator who used a semistructured interview approach to explore the group participants' beliefs and attitudes about subjects of interest to the study. Difficulty recruiting young people (aged 20 and under) to interviews or focus groups led to three groups being organised specifically with Bangladeshi youth workers in order to explore issues for younger people via the people who work most closely with them. Interviews and focus groups were recorded on audiotape and transcribed by one of the researchers.

\section{Gender and language in the interviews and focus groups}

The project research assistant (AM) is fluent in Bengali and Sylheti (a regional dialect of Bangladesh spoken by many people in Tower Hamlets). Interviews and focus groups took place in the language of preference specified by participants. A female researcher (AM) conducted all one to one interviews irrespective of the gender of the participant. Where the focus groups comprised wholly male members this was facilitated by a male researcher with appropriate language skills.

\section{Analysis}

Where interviews were conducted in Bengali or Sylheti they were translated into English before analysis. Transcripts of interviews and focus groups were analysed using interpretative phenomenological analysis, ${ }^{18}$ a systematic method for extracting themes from qualitative data widely used in health research. Two researchers were involved in this process to compare emerging themes and to identify contradictory or problematic ideas in the analysis.

\section{Ethics}

The study was approved by East London and the City Health Authority research ethics committee. Signed consent was obtained from all participants.

\section{RESULTS}

\section{Participants}

A total of 58 people were interviewed for the study, 12 in individual interviews and the remainder in six focus groups. Demographic characteristics of interview participants and composition of focus groups are detailed in tables 1 and 2, respectively.

\section{Main themes}

Four main themes were reported as impacting on access to services: confidentiality concerns, relevance of services to the community, problems with discussing sexual issues, and problems with previous experiences of health promotion. Community values regarding sex outside of marriage were an important underlying factor in participants' response to most of the questions. Sex outside of marriage was universally reported as "haram" (forbidden by Islamic teaching) and something that would bring shame and social stigma on the individual if this became known in the community.

\section{Confidentiality}

Participants consistently identified worries about confidentiality as a barrier to access. This was a consistent finding

Table 1 Demographic characteristics of interview participants

\begin{tabular}{lll}
\hline & Male $(\mathbf{n}=\mathbf{7})$ & Female $(\mathbf{n}=5)$ \\
\hline $\begin{array}{l}\text { Median age (years) } \\
\text { Range }\end{array}$ & 28 & 27 \\
Employment & $21-42$ & $20-52$ \\
$\begin{array}{l}\text { Working } \\
\text { Housekeeper }\end{array}$ & 5 & 3 \\
Unemployed & 0 & 2 \\
Education & 2 & 0 \\
$\begin{array}{l}\text { University } \\
\text { Secondary }\end{array}$ & 2 & 1 \\
Primary & 5 & 3 \\
Place of birth & 0 & 1 \\
Bangladesh & & \\
UK & 6 & 4 \\
\hline
\end{tabular}




\begin{tabular}{|lllll|}
\hline Table 2 & Composition of focus groups (FG) & \\
\hline & $\begin{array}{l}\text { Number of } \\
\text { participants }\end{array}$ & Age (median years) & Sex & Description \\
\hline FG1 & 7 & 28 (range 23=39) & Female & Community group \\
FG 2 & 12 & 13.5 (range 13-17) & Female & Youth group \\
FG 3 & 5 & 24 (range 21-29) & Female & $\begin{array}{l}\text { Youth workers } \\
\text { FG 4 }\end{array}$ \\
& 3 & 24 (range 23-26) & 2 male & Youth workers \\
FG 5 & 14 & 40 (range 32-60) & Female & Community group \\
FG 6 & 5 & 32 (range 25-45) & Male & Youth workers \\
\hline
\end{tabular}

across gender and age groups and between professionals and non-professionals. There was little or no awareness that sexual health records were maintained separately from other medical records, that GPs were not routinely notified of results, or that health professionals had a duty to maintain the confidentiality of their clients.

- "Like on the form they ask a question-do you want your GP to know and I said no way. He's one of those doctors who goes around telling stories. He's Bangladeshi and friendly as well so he's talking to everyone, telling everyone's business." (Male 21)

Many young men and women in interviews and focus groups thuoght that to be seen accessing the service by another member of the Bangladeshi community would have a considerable impact on their standing in the community. This issue was less important among older participants although was still raised by a number of people. One participant illustrated how this might impact on marriage prospects in the future.

- "If the older generation found out that someone was having an STD test, the whole area would find out so they would be saying don't give your daughters away to him." (Male 21)

\section{Relevance of services}

Many older participants, particularly women, thought that sex outside of marriage did not occur or that if it did it was a rarity and that therefore sexual health issues were not a relevant or important issue for the Bangladeshi community.

- "We don't really have cause to discuss these things, they don't affect us." (Female 40)

- "Women learn after marriage. We're Bengali Muslims, before marriage girls don't do anything bad, they don't have boyfriends, they don't go out with boys." (Female 52)

- "It's all about sleeping around and that doesn't really apply to us." (Focus group 3)

Younger people and participants in professionals' focus groups acknowledged that sex outside of marriage did occur and that there was a need for improved access to sex education and information about STIs/HIV. There was no gender difference among this group. Some participants reported that sex education, including outreach work and health promotion, was un-Islamic and haram.

- "For young people it's forbidden. Teaching them is forbidden and finding out forbidden. When they come to the right stage in their lives, they will find out naturally. When they are married ,they'll find out by themselves (laughs)." (Female 40)

\section{Discussing sex}

Difficulties in discussing sexual issues were highlighted by a number of participants. Traditionally grandmothers or a sister in law would be expected to take a role in educating Bangladeshi women about sexual issues before marriage.

- "Before a girl gets married they will get a grandmother or sister in law to explain everything. How it happens, how it doesn't happen, what to do, what not to do...regarding sex." (Focus group 5)

However, for many Bangladeshis in the United Kingdom this advice might come several years after they have become sexually active and would not include information about STIs. Men were reported as being more likely to get their sexual knowledge from peers; however, many said that information gained was often partial, unclear, or unhelpful. Experience of sex education was reported as being very variable, with schools seldom a source of useful information.

- "I remember having sex education but I found it quite confusing and didn't really understand." (Male 28)

Some participants felt that it was haram to teach sex education to young people and reported that parents might choose to have their children opt out of this at school. GPs were reported as a common source of information among older Bangladeshis while non-Bangladeshi friends and media were a common source of information among younger Bangladeshis irrespective of gender. The "shyness" of Bangladeshis regarding this issue was a consistent theme, as was the idea that to discuss these issues with someone implied a lack of respect for them.

- "When we were watching TV with my family, whenever there was kissing on the programme we had to change channel." (Focus group 1)

- "I think when you look at things like sexual advice and all that, people tend to feel that it's like bringing shame to yourself." (Male 24)

Sex and age of clinicians was also a consistent theme in the analysis. It was reported that it was inappropriate in Bangladeshi culture to be examined by or discuss sexual issues with someone of the opposite sex or where the clinician was of a younger generation, although some participants acknowledged that this may be less of an issue with some second generation patients.

- "A woman went to her GP with this problem and took her daughter with her. When she got there, the doctor was a man. She couldn't open herself up in front of him so she came back home." (Focus group 3)

The availability of same sex clinicians in the clinic was reported as desirable by a majority of participants, a finding validated by the steering group. The need for age appropriate 
clinicians was not suggested by participants or the steering group suggesting that this is a less pressing issue.

\section{Health promotion and sex education}

Previous culturally inappropriate attempts at sexual health promotion were a subject discussed by most community workers in focus groups.

- "Health promotion came along and gave out condoms and sexually explicit leaflets to the girls, when their parents saw this they were forbidden from coming to the youth club again." (Focus groups 3, 4, 6)

Focus groups reported that the local hospital was perceived as culturally insensitive and antagonistic towards community values. This impression was also confirmed by the steering group. The potential for harmful interventions was raised by several agencies as was wariness about engaging with sexual health services in case it damaged their standing within the community.

- "If you give out condoms it's like saying they're allowed to do it safely when our culture says no sex before marriage." (Focus group 1)

The need to balance community values with public health issues was articulated in focus groups and the steering group. There was a great deal of interest in gaining access to further information on sexual health and HIV among interview and focus group participants.

Community values regarding sex outside of marriage Community beliefs about sex outside marriage had a considerable impact on how the need for sexual health services was understood.

- "In Muslim families it is not expected to even have a boyfriend never mind be sleeping around with somebody." (Focus group 2)

- "We're Bengali Muslims, before marriage, girls don't do anything bad, they don't have boyfriends, they don't go out with boys." (Female 52)

Although many participants acknowledged that sex outside of marriage did happen the impact on families and individuals in terms of the shame and stigma that disclosure brought was a consistent theme in determining service use and how health promotion could be affected.

- "I mean of course it (sex outside of marriage) is happening but nobody talks about it because of what that would mean for your family." (Male 21).

\section{DISCUSSION}

Sexual health services need to reflect the prevailing cultural norms of the populations they serve. For the Bangladeshi community of Tower Hamlets this means acknowledging the importance of spiritual values in determining attitudes to sex outside of marriage and pervasive issues of shame and stigma associated with transgressing these community norms. These values and attitudes may prevent members of the community from accessing sexual health care. Sexual health strategies, including raising the profile of services available in the community or promoting preventative measures, are likely to have only moderate success if they do not take account of these factors and may inadvertently have a harmful effect on the sexual health of the community by discouraging attendance if inappropriately developed. This research highlights the complex social and psychological factors that underpin engagement with sexual health services. The degree of cultural expertise needed by a service to negotiate these factors is considerable and unlikely to be developed without working with community based agencies and organisations.

The majority of research issues relating to ethnicity and sexual health have looked at vulnerable or less powerful groups in society. ${ }^{19}$ A power imbalance exists where such groups may feel threatened, have little influence over the research process, or control over the impact of the results on their community. Previous studies have utilised community participation in work on reproductive health to guard against these possibilities. ${ }^{20}$ Here we used a participatory research model to develop research agendas and methodologies in collaboration with the Bangladeshi community of Tower Hamlets. It also utilises community expertise in developing and implementing recommendations arising from the research. We do not assume homogeneity in this group nor do we seek to compare our sample with other groups or to claim that it is representative of the wider Bangladeshi community. We do however seek to give our participants a voice in highlighting some of their difficulties in accessing local sexual health services.

\section{Methodological issues}

The use of qualitative methods to investigate issues of access to sexual health services allows researchers to distinguish between what a community says it does, what it actually does and the underlying beliefs that shape those ideas and behaviours. ${ }^{21}$ Our methodology used existing community networks and expertise to steer research, thus ensuring that the research agenda served both clinicians and the community. The steering group facilitated this process as it interpreted and commented upon research findings and recommendations ensuring that subsequent initiatives will be better able to meet the needs of the community. The sense of ownership of initiatives among community leaders facilitated collaborative approaches and also allowed community members to directly communicate requests for services or support to hospital trust staff in a formal setting. It was felt that the group should continue past the study and meet on a quarterly basis in order to continue the process of community involvement in developing sexual health services. We believe that this model could be widely applied to services working with culturally sensitive issues with traditionally hard to reach communities; however, the considerable work and time required to gain the trust and support of community groups should not be underestimated.

Despite good community links, recruitment of young people to the study was extremely difficult. Youth workers were recruited as a way of gaining information about the needs of younger members of the community but we acknowledge that this is far from ideal. Further work needs to address ways of engaging young people in such studies. Similarly, it proved difficult to recruit men to the focus groups, except where groups were made up of or included male youth workers. The steering group thought that this problem was not surprising as men would be more likely to find discussing issues of sexual health with peers in a public setting shameful and thought that women were more used to discuss these issues, and health problems in general, with one another. It was necessary to increase the number of men recruited to the individual interviews to compensate for this.

Our model does raise issues about who is privileged to speak on behalf of which communities. It is important to consider that elders, community leaders, or professionals working with community groups might be unrepresentative of marginalised groups within that community-for example, younger people, whose sexual values and behaviours are considered "deviant" by other members of the community. Efforts should be made to represent a wide range of views 
and be aware of viewpoints that may not be expressed within a group setting. This is particularly important when the needs of women, younger people, or people in same sex relationships are being addressed. These groups are relatively powerless within the community, which may be reluctant to acknowledge that their particular needs even exist, and may find it difficult to articulate their own needs because of social stigma.

\section{Implications of the study findings}

Services working with culturally diverse communities should harness the expertise and local skills and knowledge of existing networks of non-statutory organisations already working with those communities. These networks can provide useful routes into the services for community members who are either unaware of the service or reluctant to seek help. They can also help develop appropriate health promotion strategies. Supporting existing organisations to develop their own sexual health initiatives and materials will mean that health promotion and awareness work can begin as cultural expertise and and credibility is developing. Examples might include leaflets on existing sexual health services in an appropriate language and advertisements in local community newspapers or on local radio ("Ramadan Radio").

Working with community groups in order to utilise existing relationships of trust and credibility that have been built up over many years may be the most effective way to develop services and health promotion initiatives in many instances. New community based projects should be properly funded and supported by training and consultation work from clinical staff in the sexual health service.

Collaborations with local centres of worship can be useful ways of making information available to members of the community who are not literate via sermons, discussion groups or referral through the pastoral care staff providing care is taken to make these services acceptable to the values of the local community. Care should be taken that community leaders or professional staff are not privileged with the position of speaking for everyone in that community. The diversity of needs and opinions linked to age, gender, sexuality, and degree of acculturisation must be taken into account.

The recommendations of this research have already translated into changes to sexual health service provision locally. Clear statements about confidentiality are now provided in Bengali and educational materials which are appropriate for the Bangladeshi community are in development. Two specialist Bangladeshi health advocate posts have also been established in addition to recruitment of a health adviser of Bangladeshi origin.

\section{ACKNOWLEDGEMENTS}

We thank the East London Mosque, Brick Lane Youth Development Association (BLYDA), Social Action for Health, The Bangladeshi Drug Project (NAFAS), the University of East London, the Tower Hamlets PCT Health Advocacy Service, Barts and the London NHS Trust Health Advocacy Service, the London East AIDS Network (LEAN), Account3 Women's Consultancy Service, Dame Collet House Community Centre, ELITE Youth, and Haileybury Centre Youth Action Scheme.

\section{CONTRIBUTORS}

JP and CSE conceived the study; $\mathrm{AB}$ and $\mathrm{AM}$ were responsible for design of interview guides recruitment, interviewing, and focus group facilitation under the supervision of JP and CSE; all authors analysed data and contributed to preparation of the manuscript.

\section{Authors' affiliations}

A Beck, A Majumdar, J Petrak, Department of Psychology, The Ambrose King Centre, Royal London Hospital, London, UK

C Estcourt, Institute of Cell and Molecular Science, Barts and the London Queen Mary's School of Medicine and Dentistry, and Infection and Immunity, Barts and the London NHS Trust, London, UK

Source of funding: This project was fully funded by a grant from Barts and the London NHS Trust and Tower Hamlets PCG Interface Research Programme.

Conflicts of interest: None.

\section{REFERENCES}

1 Department of Health. National strategy for sexual health and HIV 2001 London: Her Majesty's Stationery Office, 2001.

2 Her Majesty's Stationery Office. Race Relations (Amended) Act. London: HMSO, 2000 (accepted 14 March 2003).

3 Low N, Daker-White G, Barlow D, et al. Gonorrhoea in inner London: results of a cross sectional study. BMJ 1997;314:1719.

4 Lacey CJN, Merrik DW, Bensley DC, et al. Analysis of the sociodemography of gonorrhoea in Leeds, 1989-93. BMJ 1997;314:1715.

5 DeCock KM, LowN. HIV and AIDS, other sexually transmitted diseases, and tuberculosis in ethnic minorities in the United Kingdom: is surveillance serving its purpose? BMJ 1997;314:1747.

6 Tod AM, Read C, Lacey A, et al. Barriers to uptake of services for coronary heart disease: qualitative study. BMJ 2001;323:214-17

7 Farooqi A, Nagra D, Edgar T, et al. Attitudes to lifestyle risk factors for coronary heart disease amongst South Asians in Leicester: a focus group study. Family Practice 2000;17:293-7.

8 Gott M, Hinchliff S. Barriers to seeking treatment for sexual problems in primary care: a qualitative study with older people. Family Practice 2003;20:690-5.

9 Elam G, Fenton K, Nazroo J, et al. Exploring ethnicity and sexual health. London: National Centre for Social Research, 1999.

10 Fisher JA, Bowman ML, Thomas T. Issues for South Asian Indian patients surrounding sexuality, fertility and childbirth in the US health care system $J$ American Board of Family Practice 2003; 16:2.151-155.

11 Aral SO. Understanding racial-ethnic and societal differences in STIs. Sex Transm Infect 2002;78:2-4.

12 De Silva P. Culture and sex therapy. Sexual \& Marital Therapy 1997; 14:105-7.

13 Greater London Authority. Health in London, 2002 review of London health strategy high level indicators. London: GLA, 2002.

14 Skinner CJ. Saulsbury NKG. Goh BT. Sexually transmitted infections in Bangladeshis resident in the United Kingdom: a case control study. Sex Transm Infect 2002;78:120-2.

$15 \mathrm{Kaj}$ J, Hedges C. Minority community ethnic participation in needs assessment and service development in primary care: perceptions of Pakistani and Bangladeshi people about psychological distress. Health Expect 1999;2:7-20.

16 Leighton $\mathrm{AH}$, Hughes JM. Culture as causative of mental disorder. Millbank Memorial Fund Quarterly 1961;39:446-70.

17 Fernando S, Ndegwa D, Wilson M. Forensic psychology, race and culture. London: Routledge.

18 Smith JA. Beyond the divide between cognition and discourse: using interpretative phenomenological analysis in health psychology. Psychology \& Health 1996;2:261-71.

19 Elam G, Fenton K. Researching sensitive issues and ethnicity: lessons from sexual health. Ethnicity and Health 2003;8:15-27.

20 Mullings L. Qualitative methodologies and community participation in examining reproductive experiences: the Harlem Birth Right Project. Maternal and Child Health Journal 2001;5:85-93.

21 Hall ET. Beyond culture. New York: Anchor Books, 1977. 